\title{
Research on Job-shop Scheduling Algorithm of Single Piece and Small Batch MES
}

\author{
Huangtao $^{1}$ and Tan yanna ${ }^{2}$ \\ 1. Harbin University of Science and Technology, Harbin, China \\ 2. China United Network Communications Corp Harbin branch, Harbin, China \\ Email:ht1124@163.com
}

\begin{abstract}
MES job shop scheduling problem is the core module, and its essence is a kind of resource constraints, time constraints and process constraints such as combinatorial optimization problems, research and application of job shop scheduling problem for China's manufacturing sector improve management, productivity, and the implementation of advanced manufacturing strategies are important, therefore, job shop scheduling research has theoretical and practical significance. In this paper, the slow evolution of GA algorithm will occur, or premature, and the SA algorithm is introduced to the crossover and mutation, genetic simulated annealing algorithm is proposed - GASA algorithm, mathematical model is given algorithm, the algorithm model, the algorithm process. GA parallel sampling of the time optimization algorithm performance can be improved, and the control of SA binding guidelines to control the convergence of the algorithm to avoid prematurity. The classical scheduling problem FTO6, GASA correctness of the algorithm is verified and compared with the traditional GA algorithm is verified GASA efficiency of the algorithm.
\end{abstract}

Keywords: Manufacturing execution system, shop scheduling, genetic algorithms, simulated annealing algorithm

\section{Introduction}

Researching the problems of operations planning and scheduling can promote genetic algorithms, heuristics, simulated annealing algorithm and other methods to further optimize and even mutual integration, but also providing a method for solving a similar problem similar areas [1]. Based on the analysis of traditional job-shop scheduling problem and the combination of small single batch scheduling constraints, in view of the evolution of GA algorithm will happen slowly or premature, the SA algorithm is introduced into the crossover and mutation[2], genetic simulated annealing algorithm and GASA hybrid scheduling algorithms is proposed. The scheduling algorithm can effectively enhance resource utilization, increase productivity for the enterprise to bring greater competitiveness and economic efficiency.

\section{The Description of Single and Small Batch Job-Shop Scheduling Problem}

Single and small batch manufacturing is a typical discrete manufacturing, by changing the physical shape of the raw materials, assembled to form the product. It still occupies a very important position in our state-owned enterprises [3], such as one of ships, bridges, aircraft and other manufacturing enterprises, but also a wide range of corporate form is one of the key sectors of our economy to increase production. Therefore, multi-species, single and small batch manufacturing is the future development of the overall trend, which processes shown in Figure 1. 


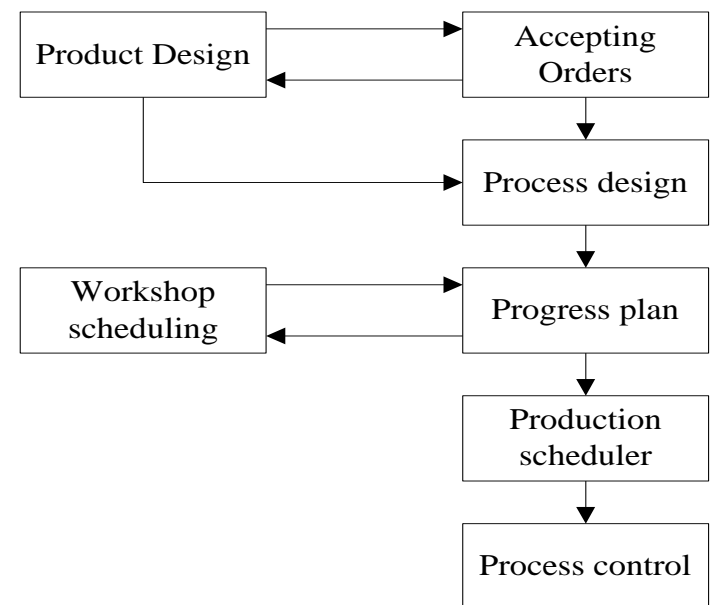

Figure 1. Operations Flow of Single Piece and Small Batch Manufacturing

Because of the complex structure of products, diversification of the production process, the process route is flexible and the production cycle is long, the degree of the specialization of production is low, a large number of uncertainties is existed in workshop manufacturing process (such as a single piece and small batch: equipment failure, order changes), resulting in difficulties in production planning and estimating, job scheduling, and control complex. Thus, the solution MES in single and small batch job scheduling problem is to determine the success or failure of production planning and control business. However, the current job scheduling algorithms mostly stay in the theoretical research, without sufficient consideration of job scheduling and product design, logistics, scheduling and other factors [4], job scheduling algorithms cannot be effectively embedded into a single piece of MES in small batches.

Due to the variability of single and small batch, resulting in that production scheduling optimization objectives cannot be unified (such as equipment operation rate was the highest product delivery schedule, the shortest total production process, etc.,) but until now, no one algorithm can be a variety of performance indicators[5], due to plant production process is not fixed, dynamic, common scheduling system is difficult to meet the actual production targets Therefore, the main idea of this article is job-shop scheduling system: First analysis of existing targets for optimization and design, optimum scheduling algorithm; Second part of the initial sorting process, while Gantt chart shows the scheduling results [6], and give full play to wisdom and experience ,dispatchers by scheduling result of human-computer interaction is adjusted to obtain optimum results.

\subsection{The Mathematical Model of Single Piece and Small Batch Scheduling Problem}

For a single-piece and small batch job shop scheduling problem, we know that each work piece order on each machine and the processing time constraints of each operation, requiring to determine the start time for each work piece machining or processing order or completion time on each machine, and finally to some indicators of production operations (such as completion time of all machines, the delay time of the work piece, the work piece flow time and production costs, etc.,) to achieve the optimum.

Single job shop scheduling problem, the flow of parts due process cannot be determined in advance, so $i, j, m$ is a description of a process of three parameters. Where $i$ represent the piece number, $j$ is a work number, $m$ represents the 
completion of the first piece $\mathrm{i} j$ processes of machine number, common to the work piece machining data is:

1. $T$ represents the processing time matrix. $T(i, j)$ representative of the work piece $\mathrm{i} j$-th step of processing time in the machine.

2. Said machine sequence matrix $J_{M}, J_{M}(i,$.$) i represents all work pieces$ arranged in the respective step of the machine according to the priority when the number of machining, $J_{M}(i, j)$ represents the $\mathrm{j}$-th completion of the work procedure i machine number.

3. $M_{J}$ represents work piece permutation matrix, $J_{M}(i,$.$) representative of the$ work sequence of the work piece in order to machine i, $J_{M}(i, j)$ representative of the machine work piece number i $\mathrm{j}$-th processing.

4. $C_{i j}$ represents the work piece on the machine $\mathrm{i} \mathrm{j}$ is complete.

Work piece permutation matrix is a representation of the scheduling Gantt chart commonly used in construction. Technical constraint matrix is machine order matrix, and processing time matrix, as is known in advance. Since the machine has the sequence order of the respective steps of the work piece machining and the work piece are restricted by the constraints of the process, so that the work piece and the machine must be met: the order in which each step of the work piece is fixed, and one or a plurality of steps to form a work piece. Each process must be processed on the specified machine, and must be processed in order to begin a process after it before processing is completed. The duration of each work piece machining process route and process fixed. A piece cannot be processed on different machines; a machine cannot be processed work piece two or more. After each process began, not interrupted or stopped midway.

\section{GASA Hybrid Scheduling Algorithm}

Due to the uneven distribution, small probability mutation, crossover evolutionary capacity is insufficient; these cases will not increase the diversity of population. So when the convergence criteria are not good, GA algorithms will evolve slowly and precocious phenomenon will occur. The SA algorithm can accept a limited degree of "bad" solution, with a simple principle, the use of flexible, etc., it can jump out of the local optimal solution, to find the global optimal or near-global optimum solution, but the SA algorithm is dependent to the annealing temperature process, and its global convergence conditions for withdrawal temperature requirements are very demanding, so the time performance of SA algorithm is bad, if the two algorithms is combined, the use of GA parallel sampling time performance improved algorithm, the SA control criterion can control the convergence of the algorithm in combination, to avoid premature. So in this paper the annealing algorithm and genetic algorithm is combined, a new single-piece and small batch job-shop scheduling optimization algorithm is proposed - genetic simulated annealing (GASA) algorithm.

MES for single and small batch job shop scheduling features, generally requiring each batch processing tasks to complete as soon as possible, so this algorithm is to minimize the time to complete the process for scheduling objectives, design GASA hybrid algorithm, the specific method is:

GASA algorithm is described as follows:

Algorithm parameters: number of populations $P_{\text {size }}$, the initial value of iteration k $=0$, the maximum number of iterations $k_{\max }$, temperature attenuation coefficient

1. The initial population is generated according to the appropriate method. 
2. Evaluation group $\mathrm{p}(\mathrm{t})$ of the fitness, if termination condition is satisfied, the end.

3. Individual crossover operation and individual variation operation.

4. Simulated annealing operation, the formation of new individual. .

On the following iterative steps L times:

(1). Generated in the neighborhood of the current solution

(2) Calculate the temperature coefficient of the adaptive decay

(3) By a temperature control function to get the current temperature T.

(4) The use of acceptable probability formula to calculate the probability of a neighborhood solution instead of the current solution.

5. Evaluate the fitness of the new individual.

6. Individual selection for the copy operation:

7. Judgment iteration termination condition. Does not satisfy the $\mathrm{k}=\mathrm{k}+1$, reversed three continue to evolve, if met, the termination of the calculation, the output current fitness strongest individuals.

\section{The Simulation Experiments of GASA Hybrid Algorithm On Job- Shop Scheduling Problem}

\subsection{A Typical Job Shop Scheduling Problem}

Given the importance and application of JSP problem, many researchers have designed a number of typical problems. Solving these problems is to test and optimize the performance comparison between the different algorithms. In this paper, the design selected by Fisher and Thompson FT06 job shop scheduling problem, the size is $6 \times 6$, the former is the number of artifacts, which is the number of machines, and each piece has six steps, have come to the optimal solution for 55 . FT06 job shop scheduling problem is a classic problem of job-shop scheduling, scheduling algorithms commonly used for detection of the feasibility and effectiveness of the tools. The initial state is as follows.

$$
\mathrm{T}=\left[\begin{array}{cccccc}
1 & 3 & 6 & 7 & 3 & 6 \\
8 & 5 & 10 & 10 & 10 & 4 \\
5 & 4 & 8 & 9 & 1 & 7 \\
5 & 5 & 5 & 3 & 8 & 9 \\
9 & 3 & 5 & 4 & 3 & 1 \\
3 & 3 & 9 & 10 & 4 & 1
\end{array}\right]\left[\begin{array}{cccccc}
2 & 3 & 1 & 4 & 6 & 5 \\
5 & 1 & 2 & 6 & 3 & 4 \\
4 & 5 & 1 & 2 & 6 & 3 \\
2 & 1 & 3 & 4 & 5 & 6 \\
5 & 2 & 1 & 6 & 3 & 4 \\
4 & 1 & 6 & 2 & 5 & 3
\end{array}\right]
$$

\subsection{FT06 Scheduling Experiments}

Convert FT06 job-shop scheduling problem into the work piece number based raw data as shown in Table 1: Table 1 FT06 scheduling problem (task number $\mathrm{i}$, the work piece number $\mathrm{j}$, the device number $\mathrm{m}$, period $\mathrm{t}$ )

Table 1. FT06's Scheduling

\begin{tabular}{|c|c|c|c|c|c|c|c|}
\hline \multirow{2}{*}{\multicolumn{2}{|c|}{$\begin{array}{l}\text { (Device number } \mathrm{m} \text {, } \\
\text { period } \mathrm{t} \text { ) }\end{array}$}} & \multicolumn{6}{|c|}{ Work number } \\
\hline & & $j=101-1$ & $j=101-2$ & $j=101-3$ & $j=101-4$ & $j=101-5$ & $j=101-6$ \\
\hline \multirow{4}{*}{$\begin{array}{l}\text { Service } \\
\text { Number }\end{array}$} & $\mathrm{i}=101$ & (A 3,1) & $(\mathrm{A} 1,3)$ & (A 2,6) & (A 4,7) & $(\mathrm{A} 6,3)$ & $(\mathrm{A} 5,6)$ \\
\hline & $\mathrm{i}=102$ & (A 2,8) & $($ A 3,5) & $(\mathrm{A} 5,10)$ & $($ A 6,10$)$ & $(\mathrm{A} 1,10)$ & (A 4,4) \\
\hline & $\mathrm{i}=103$ & (A 3,5) & $(\mathrm{A} 4,4)$ & $(\mathrm{A} 6,5)$ & (A 1,9$)$ & $(\mathrm{A} 2,1)$ & (A 5,7) \\
\hline & $i=104$ & (A 2,5) & (A 1,5$)$ & (A 3,5) & (A 4,3) & (A 5,8) & $(\mathrm{A} 6,9)$ \\
\hline
\end{tabular}




\begin{tabular}{l|l|l|l|l|l|l|l}
\hline & $\mathrm{i}=105$ & (A 3,9) & (A 2,3) & (A 5,5) & (A 6,4) & (A 1,3) & (A 4,1) \\
\cline { 2 - 8 } & $\mathrm{i}=106$ & (A 2,3) & (A 4,3) & (A 6,9) & (A 1,10) & (A 5,4) & (A 3,1) \\
\hline
\end{tabular}

After computing the resulting Gantt chart as Figure 2 below:

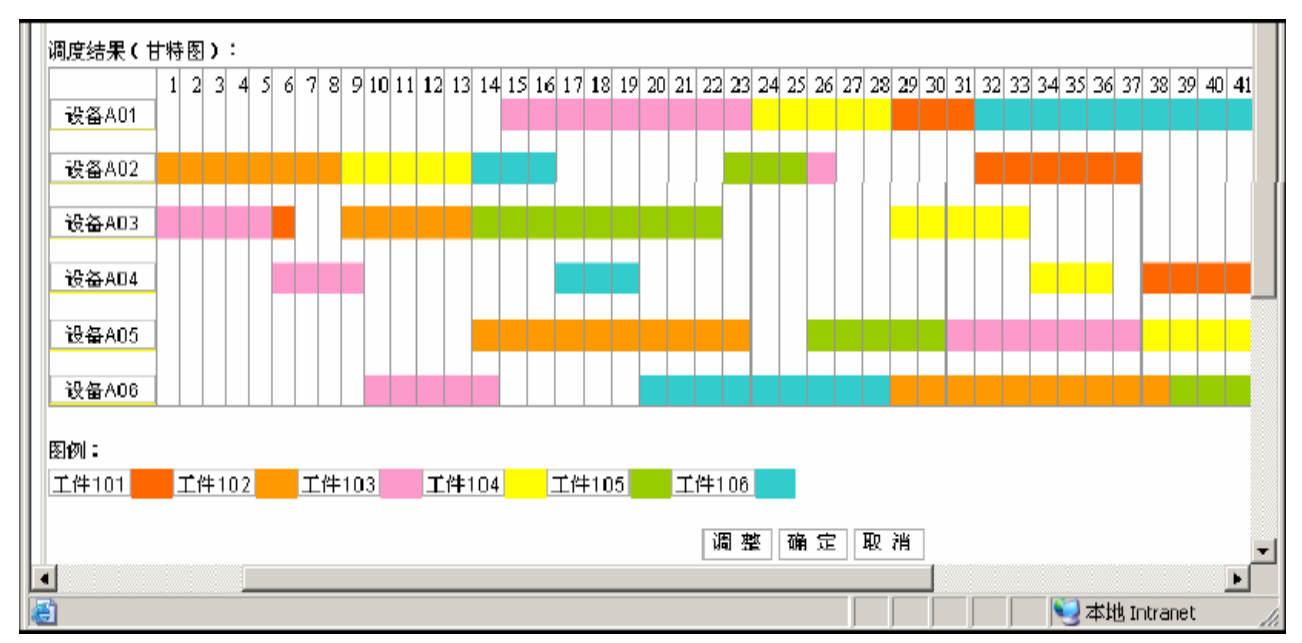

Figure 2. The Scheduling Gantt

From the Gantt chart, step 2 will be the last piece to complete the final process time is 59; this data is already very close to the optimal solution 55 .

Table 2. FT06 Scheduling Result Data

\begin{tabular}{|c|c|c|c|c|c|c|}
\hline Task i & Process $\mathrm{j}$ & $\begin{array}{l}\text { Equipment } \\
\mathrm{m}\end{array}$ & $\begin{array}{l}\text { Time } \\
\text { line } \mathrm{t}\end{array}$ & $\begin{array}{l}\text { Starting } \\
\text { time }\end{array}$ & $\begin{array}{l}\text { Ending } \\
\text { time }\end{array}$ & $\begin{array}{l}\text { Equipment } \\
\text { remaining time }\end{array}$ \\
\hline 101 & 1 & $\mathrm{~A} 3$ & 1 & 5 & 6 & 26 \\
\hline 101 & 2 & A1 & 3 & 28 & 31 & 25 \\
\hline 101 & 3 & $\mathrm{~A} 2$ & 6 & 31 & 37 & 22 \\
\hline 101 & 4 & A4 & 7 & 37 & 44 & 16 \\
\hline 101 & 5 & A6 & 3 & 44 & 47 & 9 \\
\hline 101 & 6 & A5 & 6 & 49 & 55 & 6 \\
\hline 102 & 1 & $\mathrm{~A} 2$ & 8 & 0 & 8 & 47 \\
\hline 102 & 2 & A3 & 5 & 8 & 13 & 39 \\
\hline 102 & 3 & A5 & 10 & 13 & 23 & 34 \\
\hline 102 & 4 & A6 & 10 & 28 & 38 & 24 \\
\hline 102 & 5 & $\mathrm{~A} 1$ & 10 & 45 & 55 & 14 \\
\hline 102 & 6 & A4 & 4 & 55 & 59 & 4 \\
\hline 103 & 1 & A3 & 5 & 0 & 5 & 31 \\
\hline 103 & 2 & A4 & 4 & 5 & 9 & 26 \\
\hline 103 & 3 & A6 & 5 & 9 & 14 & 22 \\
\hline 103 & 4 & $\mathrm{~A} 1$ & 9 & 14 & 23 & 17 \\
\hline 103 & 5 & A2 & 1 & 25 & 26 & 8 \\
\hline 103 & 6 & A5 & 7 & 30 & 37 & 7 \\
\hline 104 & 1 & A2 & 5 & 8 & 13 & 35 \\
\hline 104 & 2 & A1 & 5 & 23 & 28 & 30 \\
\hline 104 & 3 & A3 & 5 & 28 & 33 & 25 \\
\hline 104 & 4 & A4 & 3 & 33 & 36 & 20 \\
\hline
\end{tabular}




\begin{tabular}{c|c|c|c|c|c|c}
\hline 104 & 5 & A5 & 8 & 37 & 45 & 17 \\
\hline 104 & 6 & A6 & 9 & 47 & 56 & 9 \\
\hline 105 & 1 & A3 & 9 & 13 & 22 & 25 \\
\hline 105 & 2 & A2 & 3 & 22 & 25 & 16 \\
\hline 105 & 3 & A5 & 5 & 25 & 30 & 13 \\
\hline 105 & 4 & A6 & 4 & 38 & 42 & 8 \\
\hline 105 & 5 & A1 & 3 & 42 & 45 & 4 \\
\hline 105 & 6 & A4 & 1 & 45 & 46 & 1 \\
\hline 106 & 1 & A2 & 3 & 13 & 16 & 30 \\
\hline 106 & 2 & A4 & 3 & 16 & 19 & 27 \\
\hline 106 & 3 & A6 & 9 & 19 & 28 & 24 \\
\hline 106 & 4 & A1 & 10 & 31 & 41 & 15 \\
\hline 106 & 5 & A5 & 4 & 45 & 49 & 5 \\
\hline 106 & 6 & A3 & 1 & 49 & 50 & 1 \\
\hline
\end{tabular}

Emerged from the optimal solution of the number of iterations, the influence of parameter change on the algorithm, GASA algorithm and the traditional GA algorithm are compared. Table 3 is the two kinds of algorithms of FT06 scheduling results.

Table 3. Comparison of Scheduling Results

\begin{tabular}{|c|c|c|c|c|}
\hline Algorithm & $\begin{array}{l}\text { The optimal value in } \\
\text { algebra }\end{array}$ & $\begin{array}{l}\text { Choose } \\
\text { coefficient }\end{array}$ & $\begin{array}{l}\text { Variation } \\
\text { coefficient }\end{array}$ & $\begin{array}{l}\text { Iterative } \\
\text { optimal value }\end{array}$ \\
\hline \multirow{8}{*}{ GA } & 30 & 0.4 & 0.1 & 1442 \\
\hline & 17 & 0.75 & 0.1 & 1443 \\
\hline & 39 & 0.9 & 0.2 & 1445 \\
\hline & 9 & 0.99 & 0.1 & 1440 \\
\hline & 8 & 0.9 & 0.1 & 1440 \\
\hline & 7 & 0.9 & 0.01 & 1442 \\
\hline & 21 & 0.9 & 0.05 & 1445 \\
\hline & 31 & 0.9 & 0.15 & 1442 \\
\hline \multirow{6}{*}{ GASA } & 15 & 0.4 & 0.1 & 1445 \\
\hline & 5 & 0.75 & 0.1 & 1445 \\
\hline & 8 & 0.99 & 0.1 & 1445 \\
\hline & 8 & 0.9 & 0.01 & 1445 \\
\hline & 10 & 0.9 & 0.05 & 1445 \\
\hline & 9 & 0.9 & 0.1 & 1445 \\
\hline
\end{tabular}

For the GA algorithm, solving the problem of FT06 convergence speed is slower, convergence algebra is larger, and premature phenomenon is appeared. While GASA algorithm, solving the problem of FT06, it can converge to the optimal solution of the problem, but the genetic parameters have impact on the convergence rate. By table 3 GASA algorithm convergence optimal solutions can also be observed in 1445, in the standard genetic algorithm, the precocious phenomenon is existed, the mean fitness volatile, and converges to local optimal solution 1442; Through the operation of each generation population fitness standard deviation, it can be seen that the volatility of GASA algorithm is less than the traditional GA algorithm.

\section{Conclusions}

Based on the Job Shop Scheduling Problem theoretical research on the combination of constraints single and small batch scheduling based on genetic 
algorithm and simulated annealing algorithm on This paper presents a GASA algorithm combining the two methods, the method cited in GA SA thought, forming a variable probability SA adaptive mutation, not only prevents premature GA also enhance local and global search capabilities finally, job-shop scheduling problem using typical data sets to test the algorithm, compared with the traditional GA algorithm, GASA algorithm achieved good results.

\section{References}

[1] W. Yu, X. XIAODONG and L. Congxin, "Modeling Research on Manufacturing Execution System Based on Large-scale System Cybernetics", Journal of Shanghai Jiaotong University (Science), vol. 13, no. 6, (2008), pp. 744-747.

[2] H. Yeongdong, "The Practices of Integrating Manufacturing Execution System and Six Sigma Methodology", The International Journal of Advanced Manufacturing Technology, vol. 31, no. 11, (2006), pp. 761-768.

[3] H. Chinyin, "Distributed Manufacturing Execution Systems A Workflow Perspective", Journal of Intelligent Manufacturing, vol. 13, no. 6, (2002), pp. 485-497.

[4] A. Molina, A. R. Santaella, "Achieving E-Manufacturing Multi head Control and Web Technology for the Implementation of a Manufacturing Execution System", Journal of Intelligent Manufacturing, vol. 17, no. 6, (2006), pp. 715-724.

[5] C. Fantien, C. Chihfeng and W. Shanglun, "Development of holonic manufacturing execution systems", Journal of Intelligent Manufacturing, vol. 15, no. 2, (2004), pp. 253-267.

[6] K. S. Kulga and R. R. Gilfanov, "Integration of CAD/CAM/PDM/MES and ERP systems", Russian Engineering Research, vol. 28, no. 2, (2008), pp. 169-172. 
International Journal of $u-$ and e- Service, Science and Technology Vol.8, No. 6 (2015) 